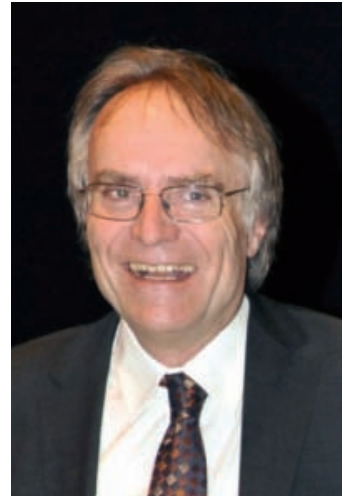

Prof. Dr. sc. nat. Beat Meier, Wädenswil

\section{Phytotherapie im Zeichen der Herausforderung}

Die Phytotherapie konnte in den letzten Monaten ihre Akzeptanz und ihr Renommee in der Schweiz in einem erheblichen Ausmass steigern. Seit 1. Juli 2011 ist das Fähigkeitsprogramm Phytotherapie (SMGP), dessen Grundkonzept schon seit bald 25 Jahren Bestand hat und sich in acht Zyklen bewährte, von der FMH anerkannt. Das Programm wurde zuletzt zusammen mit dem Schweizerischen Institut für Weiter- und Fortbildung (SIWF) modernisiert und den aktuellen Anforderungen angepasst. Rund 50 Ärztinnen und Ärzte profitieren von der Übergangsregelung und sind erfolgreiche Absolventen des Programms. Weitere werden in den nächsten Monaten und Jahren folgen, indem sie das Programm im Rahmen der Übergangsregelung abschliessen oder demnächst mit dem neuen Programm starten (Termine siehe www.smgp.ch). Die Absolventen des Programms können ab 1. Januar 2012 Phytotherapie gemäss Anhang I der Krankenpflege-Leistungsverordnung (KLV) abrechnen und dürfen sich als Phytotherapeuten bezeichnen. Die Schweizerische Medizinische Gesellschaft für Phytotherapie (SMGP) bleibt allerdings herausgefordert: Die Aufnahme in die KLV ist auf 6 Jahre befristet - eine dritte Runde zum Nachweis von Wirksamkeit, Zweckmässigkeit und Wirtschaftlichkeit (WZW) der Phytotherapie wurde damit eingeläutet. Das Bundesamt für Gesundheit ist ebenfalls herausgefordert: Endlich wurde erkannt, dass der Prozess zur Beurteilung dieser WZW-Kriterien operationalisiert werden muss. Die Eidgenössische Leistungs- und Grundsatzkommission
(ELGK) soll in Zukunft ihre Entscheide begründen und für den Antragsteller verständlich machen. Dass das bisher nicht der Fall war, tönt geradezu absurd, doch wer die Akten studiert und nach den Gründen der Ablehnung z.B. der Anträge zur Phytotherapie fragt, wird zu diesem Schluss kommen.

Mut und Engagement der SMGP sind ungebrochen. Die Gesellschaft setzt sich für die Phytotherapie als Bestandteil einer modernen Medizin im Bereich der Gesundheitspolitik sowie der Fort- und Weiterbildung ein. Sie zählt und hofft in diesen Bestrebungen auf Ihre Unterstützung.

Seit 25 Jahren ist die Schweizerische Jahrestagung für Phytotherapie der wichtigste Anlass im Fort- und Weiterbildungsprogramm. Am 17. November 2011 steht die 26. Jahrestagung mit dem Thema «Phytotherapie in der Neurologie» auf dem Programm. Das Thema überrascht, denn die Phytotherapie wird bisher kaum mit der Neurologie in Verbindung gebracht. Dabei wird deutlich, dass verschiedenste Disziplinen versuchen, Vertrauen in pflanzliche Arzneimittel zu gewinnen. Das Potenzial der Pflanzen ist nicht ausgeschöpft und wird an Bedeutung gewinnen, je weniger neue Arzneimittel, die finanzierbar sind, aus der Retorte kommen.

Die pflanzlichen Sedativa haben in den letzten Jahren viel Akzeptanz erreicht, sodass der Zeitpunkt gekommen ist, aus Sicht eines Pharmakologen deren Potenzial und die Vorteile gegenüber den synthetischen Arzneimitteln darzustellen. Vielfältig sind die Diskussionen um die Stellung der etablierten Zubereitungen aus Ginkgo biloba. Es sieht so aus, dass aus wissenschaftlicher Sicht keine Einigkeit der unterschiedlichen Meinungen bezüglich der Wirksamkeit erreicht werden kann. Der Blickwinkel der Praxis wird deshalb umso wichtiger, und die Erfahrung in komplexen Therapiesituationen muss bei der Beurteilung der Evidenz mit einbezogen werden. Inwieweit Kopfschmerzen mit Arzneipflanzen therapiert werden können, ist abzuklären.

Da die Phytotherapie in der Neurologie ein neues Fachgebiet «erobert», sind entsprechend experimentelle Felder abzustecken. Die Anwendung von Capsaicin enthaltenden Präparaten bei der Polyneuropathie ist pharmakologisch gut begründet; die Anwendung von Cannabis bleibt in der Diskussion. Zugelassene Präparate gibt es allerdings keine, da die Zulassungshürden für beide Anwendungen hoch sind. Die uns bekannten Projekte, Cannabis zur Zulassung zu bringen, sind infolge des hohen Aufwandes aus finanziellen Gründen gestoppt worden, obwohl renommierte

\section{KARGER}

Fax +497614520714 Information@Karger.de www.karger.com $1015-0684 / 11 / 0235-0248 \$ 38.00 / 0$
Prof. Dr. sc. nat. Beat Meier

Life Science und Facility Management

Zürcher Hochschule für angewandte Wissenschaften

Grüental, Postfach 335, 8820 Wädenswil, Schweiz

Tel. +41 58-9345806, Fax -9345995

beat.meier@zhaw.ch 
Firmen dahinterstanden. L-Dopa-haltige Arzneipflanzen sind aus dem asiatischen Raum bekannt und werden im Internet mit Heilversprechungen präsentiert: Was dahinter steckt und welche Empfehlungen den Patienten mit auf den Weg gegeben werden sollen, das wird - so wie alle zuvor angesprochenen Themen - an der Tagung in Baden zur Diskussion gestellt. Die Referierenden sind über die Phytotherapie hinaus ausgewiesene Experten auf ihrem Fachgebiet, sodass mit spannenden Referaten und Diskussionen gerechnet werden kann. Weil die Organisatoren von der SMGP, dem Institut für Naturheilkunde an der Universität Zürich, der Rheinburg-Klinik in Walzenhausen und der Fachgruppe Phytopharmazie an der Zürcher Hochschule für angewandte Wissenschaften in Wädenswil mit dem Thema Neuland beschreiten, bietet diese von der Schweizerischen Gesellschaft für Innere Medizin (SGIM), der Schweizerischen Gesellschaft für Allgemeinmedizin (SGAM) und der Foederatio Pharmaceutica Helvetiae (FPH) anerkannte Weiterbildung Themen, die in Lehrbüchern nicht zu finden sind. Der Weg nach Baden wird sich in vieler Hinsicht lohnen, zumal die wichtigsten Schweizer Hersteller in der die Tagung begleitenden Ausstellung einen Überblick zum phytotherapeutischen Sortiment in der Schweiz bieten. Die Veranstalter würden sich freuen, auch Sie in Baden begrüssen zu dürfen. Das Tagungsprogramm, ein Anmeldetool und Informationen zum Ort, zum Ablauf und zu den Referierenden sind zu finden unter: www.smgp.ch/auspro/jtagung/2011/tag11.html. 